\title{
The Research of Sound Absorption Performance on Micro-Perforated Panel Absorb-er
}

\author{
M. Z. SUN ${ }^{\star, a, 1}$, C. YUE ${ }^{1}$ and X. M. ZHANG ${ }^{1}$ \\ ${ }^{1}$ Hubei University of Technology, Wuhan 430068, China \\ a316460533@qq.com \\ *Corresponding author:
}

Keywords: Micro-perforated Panel Absorb-er, Absorption Performance, Structural Parameters, Absorption Coefficient.

\begin{abstract}
This paper introduces the classical theory of MPA (micro-perforated panel absorb-er). Its sound absorption coefficient primarily related to these structure parameters. Based on the theory of MPA which is proposed by M, sound absorption performances are researched by using MATLAB. By this method, the influence of different structural parameters on the sound absorption coefficient is obtained. And MPA samples are fabricated by taking typical parameters for international standard impedance tube size. The frequency ranges from $100 \mathrm{~Hz}$ to $5000 \mathrm{~Hz}$. The absorption coefficient of these samples is measured. The experimental results are in agreement with the numerical simulation results.
\end{abstract}

\section{Introduction}

The development of the perforated panel lasts for more than half a century, it is can control the highly -favored resonance characteristics, but it must use additional porous sound-absorbing material to remedy the insufficient absorption. When an aperture is smaller to a certain extent, the sound wave can effectively absorbed and does not require additional porous sound-absorbing material, which is the concept of micro-perforated panel ${ }^{[1]}$. MPA belongs to a simple resonance sound absorption structure that is made up of perforation diameter under 1mm and a cavity behind. However, an MPA sample was not proved in actual productions.

The limitations of the processing conditions and the appropriate combination of structural parameters are taken into account to design standards for sound absorption model. Assumed several groups structure parameters to simulate the relationship of the sound frequency and absorption coefficient by MATLAB and then fabricated by laser processing. The experimental data are analyzed, and the validity of the test data is verified.

\section{Classical Theory}

MPA was proposed in 1975 by the famous acoustics expert M, and the relevant theoretical model was established. According to classical MPA model ${ }^{[2]}$, Function (1) is used to calculate the relative sound impedance $^{[3,4]}$.

$$
r=\frac{0.335 t}{p d^{2}}\left[\left(1+\frac{k^{2}}{32}\right)^{\frac{1}{2}}+\frac{\sqrt{2} k \bullet d}{32 t}\right]
$$

In this function, $d(\mathrm{~mm})$ is the bore diameter, $t(\mathrm{~mm})$ is the thickness, $p(\%)$ is the perforation ratio, $k=d \sqrt{\frac{f}{10}}$ is the parameter of MPA, $f(\mathrm{~Hz})$ is the sound frequency.

Function (2) is used to calculate the relative sound quality.

$$
m=\frac{0.000294 t}{p}\left[1+\left(9+\frac{k^{2}}{2}\right)^{-\frac{1}{2}}+\frac{0.85 d}{t}\right]
$$


In this function, $m$ is the relative sound quality.

Based on Function (1) and (2), the absorption coefficient of MPA can be calculated.

$$
\alpha=\frac{4 r}{(1+r)^{2}+(\omega m-\cot (\omega D / c))^{2}}
$$

In this function, $\mathrm{D}(\mathrm{mm})$ is the back of cavity depth, $\alpha$ is the sound absorption coefficient.

Based on Function (3), when the MPA is in the resonance frequency, the absorption coefficient achieves the maximum value.

$$
\alpha_{\max }=\frac{4 r}{(1+r)^{2}}
$$

\section{MATLAB Simulation}

The sound absorption coefficient of MPA is determined by structural parameters, such as the perforation diameter, thickness, perforation rate and cavity depth. In order to obtain the influence of the structural parameters on their sound absorption characteristic, taking several groups by changing one parameter value and others constant to calculate the coefficient $[5,6]$.

\section{Perforation Diameter}

In order to study the influence of the perforation diameter on the absorption coefficient, assumed that $\mathrm{t}=0.1 \mathrm{~mm}, \mathrm{p}=3 \%$ and $\mathrm{D}=120 \mathrm{~mm}$, $\mathrm{d}$ is the only variable.
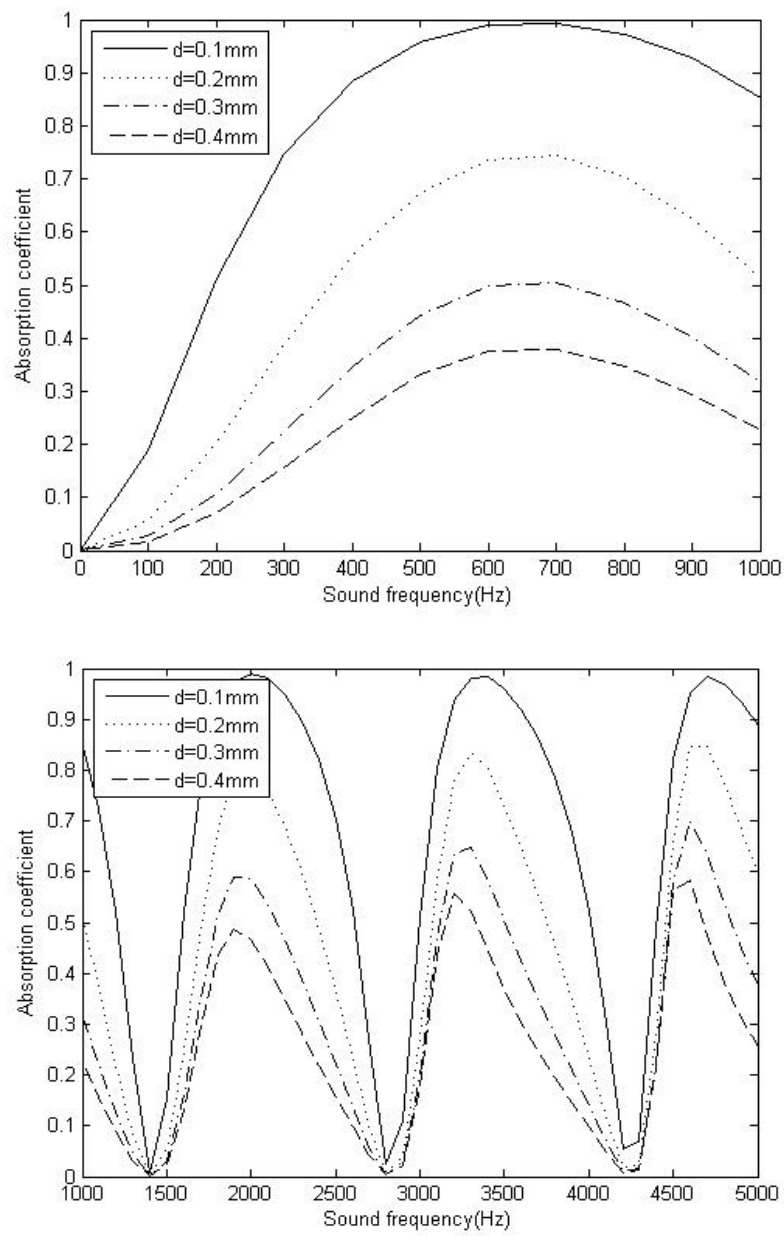

Figure1. curve of sound frequency and absorption coefficient by changing $d$ 
Simply consider the impact of the perforation diameter on the absorption coefficient. From figure 1, the sound absorption coefficient will be better when the diameter getting smaller. The maximum coefficient increase with the decrease of diameter, followed by widening of the absorption band.

\section{Thickness}

In order to study the influence of the thickness on the absorption coefficient, assumed that $p=3 \%$, $\mathrm{d}=0.1 \mathrm{~mm}$ and $\mathrm{D}=120 \mathrm{~mm}, \mathrm{t}$ is the only variable.
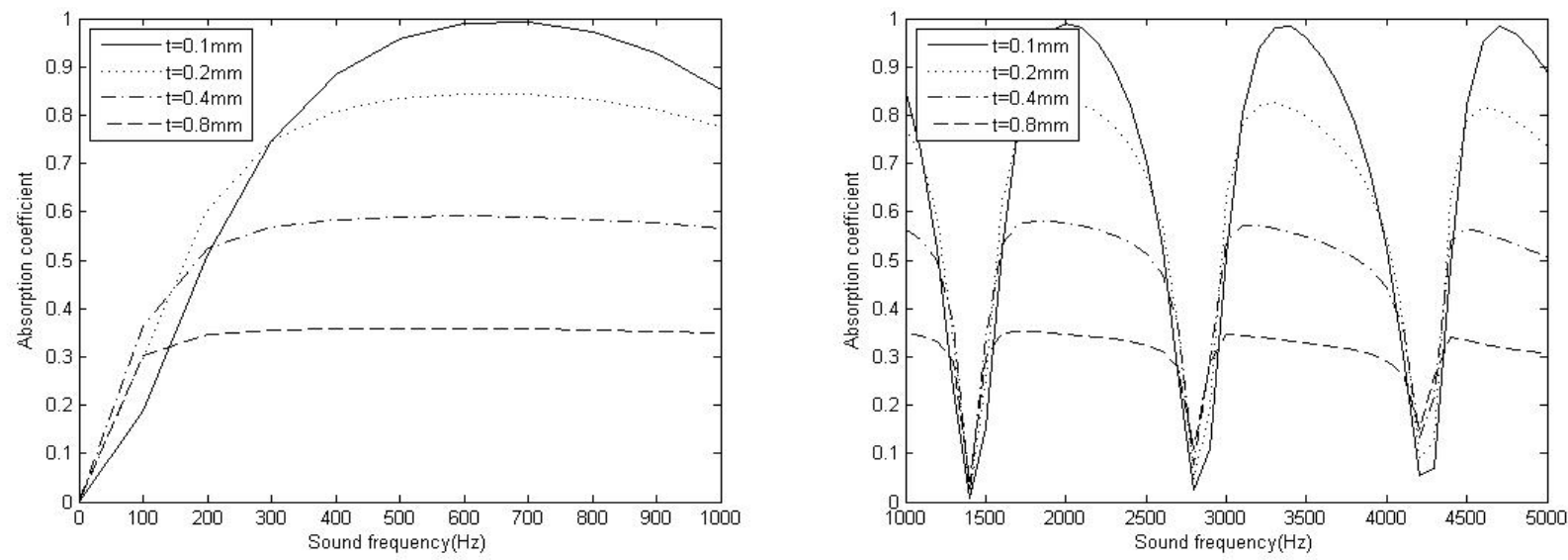

Figure2. curve of sound frequency and absorption coefficient by changing $\mathrm{t}$

From figure 2, the sound absorption coefficient will be better with the increasing of thickness when the frequency range is less than $300 \mathrm{~Hz}$. However, the higher the maximum absorption coefficient when the thickness of the thinner. But when the resonant frequency shifts to higher frequencies, maximum coefficient of frequency range decreases as the thickness increases. It is not suitable that the thickness is as small or as big as possible.

\section{Perforation Rate}

In order to study the influence of the perforation rate on the absorption coefficient, assumed that $\mathrm{t}=0.1 \mathrm{~mm}, \mathrm{~d}=0.1 \mathrm{~mm}$ and $\mathrm{D}=120 \mathrm{~mm}, \mathrm{p}$ is the only variable.

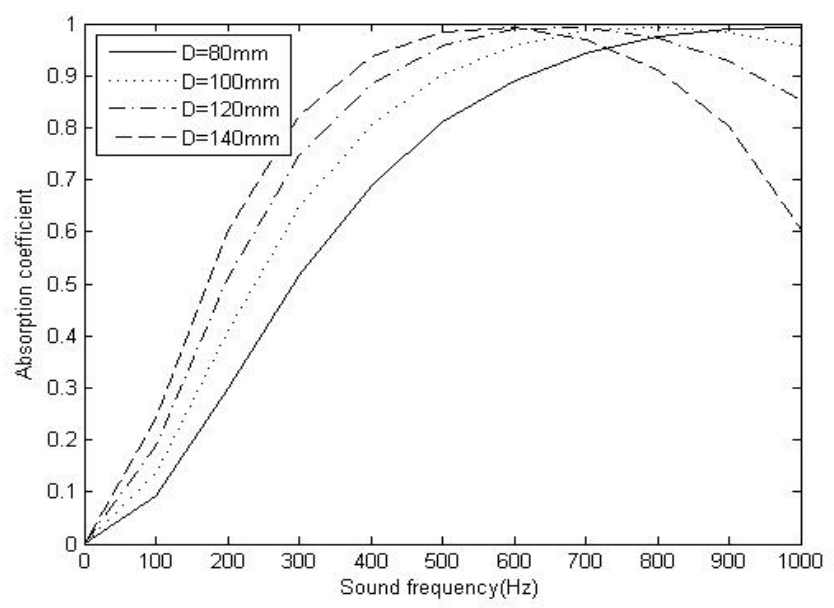




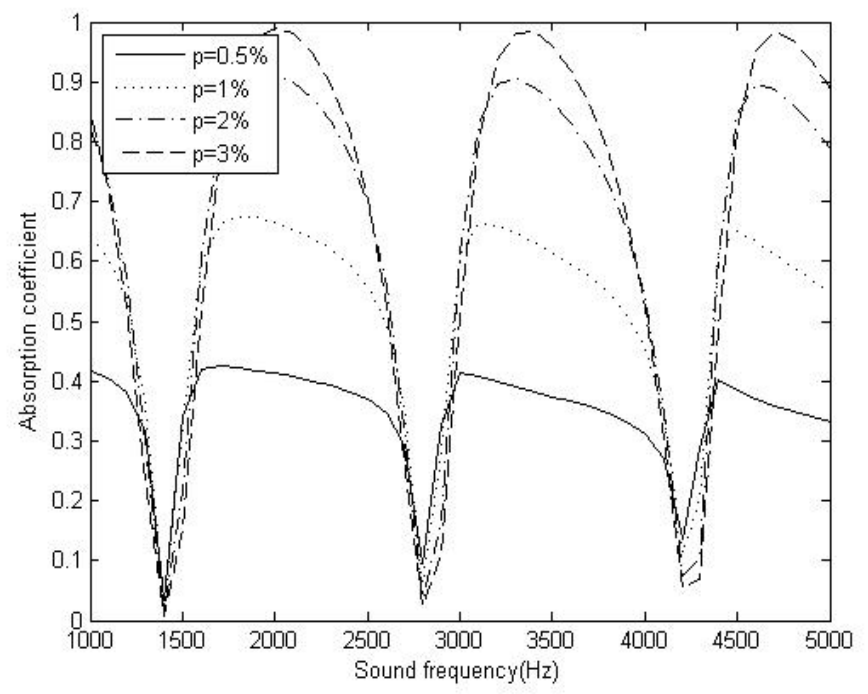

Figure3. curve of sound frequency and absorption coefficient by changing $\mathrm{p}$

From figure 3, the sound absorption coefficient will be better with the increasing of perforation rate. But the frequency range of the maximum coefficient is decreased. If the perforation rate is too large, the coefficient will be higher, but the absorption band will be smaller. If the perforation rate is too small, the absorption band will be increasing, but the coefficient will be too low. However, in practical application, the perforation rate should be chosen properly.

\section{Cavity Depth}

In order to study the influence of the cavity depth on the absorption coefficient, assumed that $\mathrm{t}=0.1 \mathrm{~mm}, \mathrm{~d}=0.1 \mathrm{~mm}$ and $\mathrm{p}=3 \%$, $\mathrm{D}$ is the only variable.

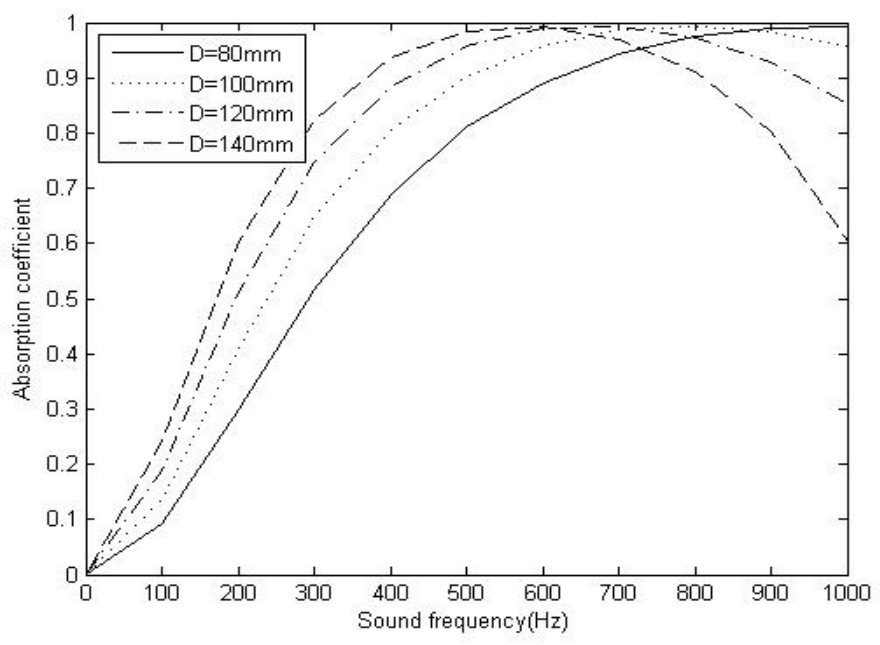




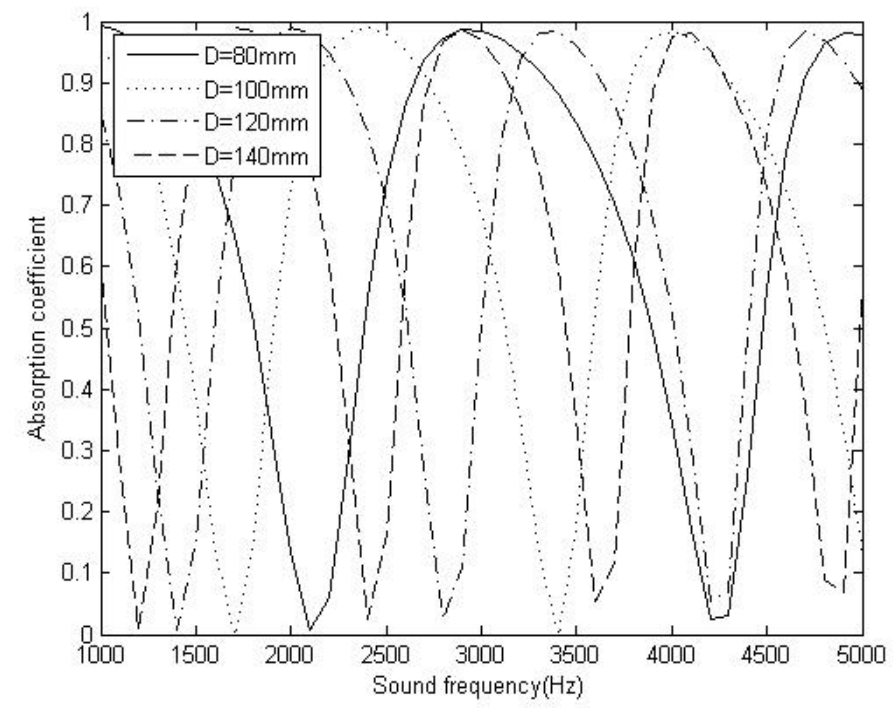

Figure4. curve of sound frequency and absorption coefficient by changing D

From figure 4, the depth of the cavity does not affect the maximum of the absorption coefficient. But the frequency coverage of maximum absorption coefficient will be larger when cavity depth is deeper.

It has been proved that sound absorption coefficient is effected by perforation diameter, thickness, perforation rate and cavity depth. When a frequency in the range of $0-5000 \mathrm{~Hz}$, the smaller the diameter of the hole is, the better the sound absorption coefficient is. But it is difficult to machine diameter less than $0.1 \mathrm{~mm}$ micro pores in actual production process. The thickness of the perforated panel is not the smaller or bigger is better. So we can confirm other structural parameter, and then chooses the appropriate structural parameter. From the simulation results, it can be seen that we can get the maximum absorption coefficient if the perforation rate is 3\%. The smaller cavity depth is, the wider the sound of the absorption band is. According to the practical situation, we should combine with the size of impedance to choose appropriate cavity depth.

\section{Experiment}

Commonly there are two kinds of measurement method of sound absorption coefficient. The one is impedance tube method and the other is reverberation chamber method. In order to manufacture the size of the impedance which meets the standard of international B\&K 4206, the samples are made into a diameter of $100 \mathrm{~mm}$ round aluminum plate. According to GB/T 18696 absorption coefficient of the sound impedance and acoustic impedance measurement, we measure the sound absorption coefficient of the samples [7]. Each sample after nine measured average. Lastly compare what we have measured absorption coefficient with the theoretical value by MATLAB Compared with theoretical values and experimental values.

\section{Experimental Data}

Take $d=0.1 \mathrm{~mm}, t=0.1 \mathrm{~mm}, p=3 \%$ and $D=120 \mathrm{~mm}$ to product micro-perforated model, carried nine times repeated experiment, respectively $A_{11}, A_{12}, A_{13}, A_{21}, A_{22}, A_{23}, A_{31}, A_{32}, A_{33}$. 
Table1 Standard deviation according to nine times repeated experiment

\begin{tabular}{|c|c|c|c|c|c|c|c|c|c|c|}
\hline \multirow{2}{*}{$\mathrm{f}(\mathrm{Hz})$} & \multicolumn{9}{|c|}{ Absorption coefficient } & \multirow{2}{*}{$\begin{array}{c}\text { Standard } \\
\text { deviation }\end{array}$} \\
\cline { 2 - 13 } & $\mathrm{A}_{11}$ & $\mathrm{~A}_{12}$ & $\mathrm{~A}_{13}$ & $\mathrm{~A}_{21}$ & $\mathrm{~A}_{22}$ & $\mathrm{~A}_{23}$ & $\mathrm{~A}_{31}$ & $\mathrm{~A}_{32}$ & $\mathrm{~A}_{33}$ & \\
\hline 100 & 0.15 & 0.12 & 0.14 & 0.13 & 0.14 & 0.15 & 0.12 & 0.11 & 0.14 & 0.01 \\
\hline 200 & 0.16 & 0.14 & 0.12 & 0.15 & 0.17 & 0.14 & 0.15 & 0.16 & 0.15 & 0.02 \\
\hline 300 & 0.10 & 0.12 & 0.11 & 0.12 & 0.14 & 0.12 & 0.1 & 0.12 & 0.13 & 0.01 \\
\hline 400 & 0.46 & 0.46 & 0.47 & 0.5 & 0.51 & 0.44 & 0.48 & 0.45 & 0.50 & 0.03 \\
\hline 500 & 0.78 & 0.79 & 0.79 & 0.78 & 0.79 & 0.78 & 0.78 & 0.78 & 0.77 & 0.01 \\
\hline 600 & 0.78 & 0.81 & 0.81 & 0.87 & 0.85 & 0.83 & 0.84 & 0.84 & 0.82 & 0.03 \\
\hline 700 & 0.98 & 0.98 & 0.96 & 0.94 & 0.96 & 0.95 & 0.98 & 0.97 & 0.98 & 0.02 \\
\hline 800 & 0.99 & 0.99 & 0.99 & 0.94 & 0.94 & 0.96 & 0.98 & 0.96 & 0.98 & 0.02 \\
\hline 900 & 0.99 & 0.99 & 0.99 & 0.99 & 0.99 & 0.99 & 0.99 & 0.99 & 0.99 & 0.00 \\
\hline 1000 & 0.98 & 0.99 & 0.99 & 0.98 & 0.98 & 0.96 & 0.96 & 0.98 & 0.98 & 0.01 \\
\hline
\end{tabular}

From table.1, it can be concluded that the absorption coefficient of the standard deviation of the experimental data is smaller and the repeat-ability of experimental data is very well by nine times repeated experiment.

\section{Compare Experimental Value with Theoretical Value}

Assumed that $\mathrm{p}$ is $3 \%, \mathrm{~d}$ is $0.1 \mathrm{~mm}$, and $\mathrm{t}$ is $0.1 \mathrm{~mm}, \mathrm{D}$ is the only variant [8].

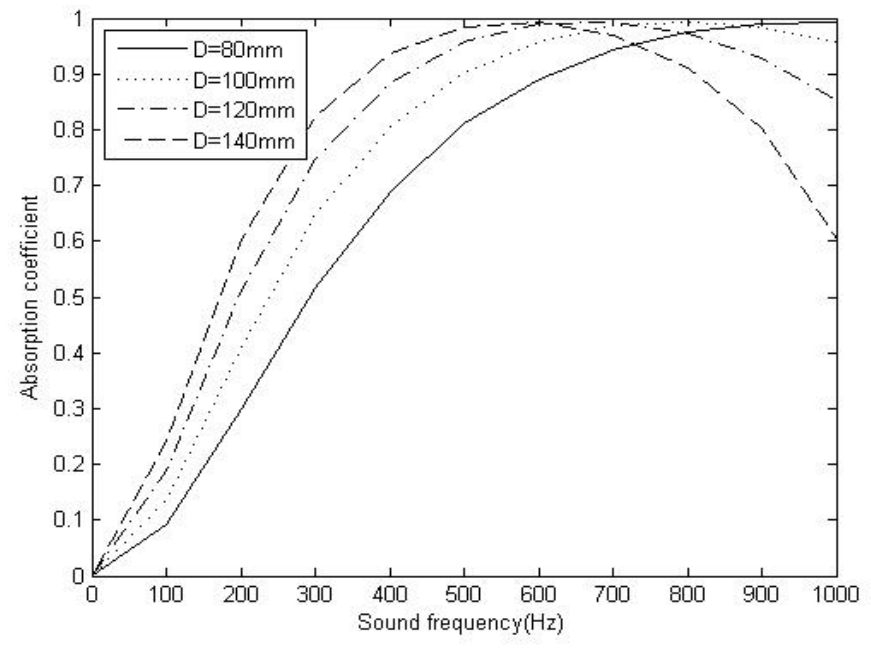

(a) Absorption coefficient curve by MATLAB calculated 


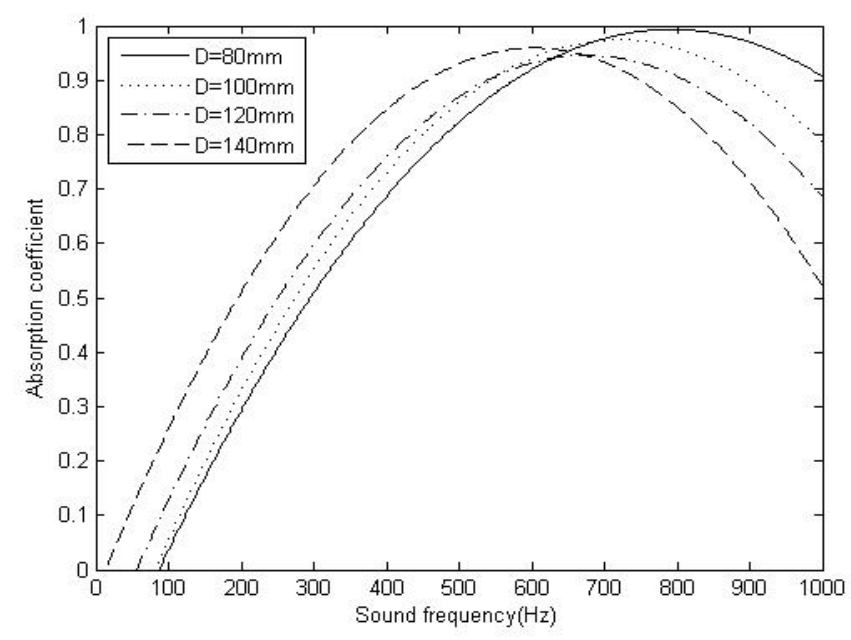

(b) Absorption coefficient curve by experimental value

Figure5. Absorption coefficient curve

By comparing the curve of experimental value and theoretical value, the two curves are not perfectly match. Apart from the structure parameters of MPA, environmental factors such as temperature, pressure, and humidity will affect the results of the experiment.

\section{Conclusions}

The classical theory model of MPA that proposed by professor M was summarized. According to the formula, the absorption coefficient can be calculated. Assumed the structure parameters of several groups and using MATLAB to simulate the curve of the sound frequency and absorption coefficient. In this way, we find that the structural parameters directly affect the sound absorption performance. Laser processing technology is used to produce MPA samples. Application of measure equipment tests the absorption coefficient of MPA. Simulation method remains to be improved by experimental research.

\section{Acknowledgments}

This work was financially supported by the Natural Science Foundation of Hubei Province of China (2014CFB178) and open foundation of State Key Lab of Digital Manufacturing Equipment \& Technology (DMETKF2014017).

\section{References}

[1] M: ‘The Modern Acoustics Theory’, Science Press, 2004.

[2] M: 'The Accuracy of the Sound Absorption of Micro Perforated Panel Body Theory and Design', Acta Acustica, 1997, 22, 385-393.

[3] Wenjuan Sun, Deyi Kong and Hui You: 'The Sound Absorption of Micro Perforated Panel Body Acoustic Performance Simulation’, Instrument Technology, 2012, 3, 10-14.

[4] Zhihui Ma and Xianhui Li: 'The Study Progress of Micro Perforated Panel Sound Absorption', Noise and Vibration Control, 2012, 10, 26-28.

[5] Penghui Liu, Yiqian Yang and Jingchuan Yao: 'Study on Absorption Property Sound-Absorbing Materials’, Noise and Vibration Control, 2011, 4, 123-126. 
[6] Peng Hu, Gongping Mao and Xiaodan Zhao: 'Effects o f Structure Parameters on the Sound Absorption of Micro Perforated Panel Absorber', Piezoelectrics and Acoustooptics, 2013, 10,624:627.

[7] Y.J.Qian, D.Y.Kong, S.M.Liu, S.M.Sun and Z.Zhao: 'Investigation on micro-perforated panel absorber with ultra-micro perforations’, Applied Acoustics, 2013, 934-935.

[8] Qingquan Hou, Yuhai Peng and Jinman Wang: 'Research on Influence Factors of Absorption Performance for Wooden Perforated Panel’, Advanced Materials Research, 2010, 1959-1963. 
